\title{
The application of a vacuum ultraviolet Fourier transform spectrometer and synchrotron radiation source to measurements of: IV. The $\beta(6,0)$ and $\gamma(3,0)$ bands of NO
}

\author{
A. S-C. Cheung, D. H-Y. Lo, and K. W-S. Leung \\ Department of Chemistry, The University of Hong Kong, Hong Kong \\ K. Yoshino \\ Harvard-Smithsonian Centre for Astrophysics, Cambridge, Massachusetts 02138
}

A. P. Thorne and J. E. Murray

Blackett Laboratory, Imperial College, London SW7-2BZ, United Kingdom

K. Ito and T. Matsui ${ }^{\mathrm{a})}$

Photon Factory, KEK, Tsukuba, Ibaraki 305-0801, Japan

T. Imajo

Japan Women's University, Tokyo 112-8681, Japan

(Received 17 July 2001; accepted 2 October 2001)

\begin{abstract}
The $\beta(6,0)\left(B^{2} \Pi_{r}-X^{2} \Pi_{r}\right)$ and $\gamma(3,0)\left(A^{2} \Sigma^{+}-X^{2} \Pi_{r}\right)$ bands of NO have been recorded using a vacuum ultraviolet Fourier transform spectrometer with synchrotron radiation as light source. The analysis of the $\beta(6,0)$ and $\gamma(3,0)$ bands of NO provides accurate rotational line positions and term values. Molecular constants of the $v=6$ level of the $B^{2} \Pi_{r}$ and $v=3$ level of the $A^{2} \Sigma^{+}$have been determined. Accurate rotational line strengths have also been obtained. The band oscillator strength of the $\beta(6,0)$ and $\gamma(3,0)$ bands are determined to be $0.48 \times 10^{-4}$ and $2.69 \times 10^{-4}$, respectively.

(c) 2002 American Institute of Physics. [DOI: 10.1063/1.1421064]
\end{abstract}

\section{INTRODUCTION}

In the wavelength region $175-205 \mathrm{~nm}$ the penetration of solar radiation into the atmosphere is controlled by the absorption cross sections of the Schumann-Runge bands of $\mathrm{O}_{2}$. Part of the radiation transmitted is available to photopredissociate NO, which has a number of strong bands with very narrow lines in the region 183-195 nm. With recent advancement of new spectroscopic techniques, we thought it would be beneficial to record and analyze these NO systems with a resolution that is comparable to the Doppler width to obtain accurate line positions and, more importantly, the band oscillator strengths of these bands. Discussions on the necessity for high resolution measurements of NO have been presented in previous papers of the series on NO bands. ${ }^{1-3}$ In these papers we describe how we combined high-resolution vacuum ultraviolet (VUV) Fourier transform (FT) spectrometry with synchrotron radiation by taking the Imperial College (IC) VUV FT spectrometer to the synchrotron radiation source at Photon Factory, KEK, Japan, where a suitable zero dispersion two-grating predisperser is available on beam line 12-B. (The predisperser is necessary to limit the bandwidth to a few $\mathrm{nm}$ in order to achieve acceptable signal-to-noise ratios, S/N.) We have used this combination of facilities to make ultra high resolution cross-section measurements of NO in the wavelength region $195-160 \mathrm{~nm}$. The present paper in this series presents the spectroscopic analysis and the

${ }^{a)}$ Present address: Tsukuba University, Ibaraki 305-8573, Japan. line and band oscillator strengths of the $\beta(6,0)$ $\left(B^{2} \Pi_{r}-X^{2} \Pi_{r}\right)$ and $\gamma(3,0)\left(A^{2} \Sigma^{+}-X^{2} \Pi_{r}\right)$ bands of NO.

An extensive review of the earlier experimental work on the electronic spectrum of $\mathrm{NO}$ molecules is given by $\mathrm{Mi}$ escher and Huber. ${ }^{4}$ The absorption spectra of NO were photographed in the VUV region for the first time by Leifson. ${ }^{5}$ He noticed the three groups of band systems, now known to be the $\gamma, \beta$, and $\epsilon$ bands. Herzberg and Mundie ${ }^{6}$ observed anomalous intensity distribution in the NO bands and assumed that the $\epsilon$ bands are a continuation of the $\gamma$ bands. Tanaka ${ }^{7}$ investigated the absorption bands of NO, and confirmed anomalous intensity in the $\gamma$ bands. He assigned the $\gamma$ bands up to $v^{\prime}=7$ by following the comments of Herzberg and Mundie. The existence of both the $\gamma$ and $\epsilon$ bands was confirmed by observation of the $\gamma(4,0)$ band in the $\epsilon(0,0)$ band by Herzberg et $a l .{ }^{8}$ However, the $\gamma(3,0)$ band has never been analyzed rotationally in absorption. The molecular constants of the $v=3$ level of the $A^{2} \Sigma^{+}$state have been available from observation in emission. ${ }^{9-11}$ Recently Danielak et al. ${ }^{12}$ reinvestigated in emission the $\gamma$ bands of NO including the $(3,0)$ band with a high resolution grating instrument. The $\beta(6,0)$ band of NO was studied extensively by Miescher and co-workers. ${ }^{8,13}$ They observed the $(5,0)$ to $(19,0)$ bands of the $\beta$ system in absorption by using a medium resolution grating instrument, and presented the rotational constant for the $B(6)$ level.

The cross section measurements of the $B(6)-X(0)$ and $A(3)-X(0)$ bands by Bethke ${ }^{14}$ were performed at low resolution, $0.04 \mathrm{~nm}$, in the presence of Ar. The rotational lines were pressure broadened to well beyond the instrumental 

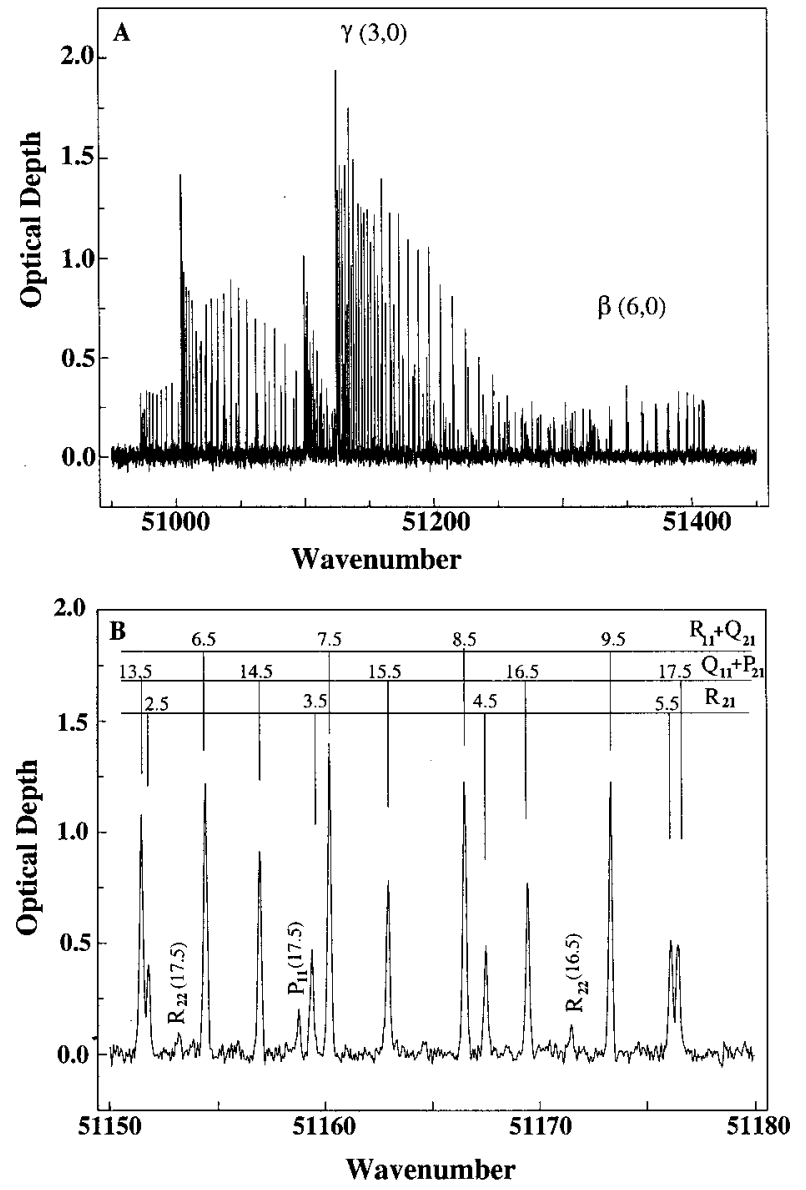

FIG. 1. The $\beta(6,0)$ and $\gamma(3,0)$ bands at 0.300 Torr of NO with $7.54 \mathrm{~cm}$ path length is shown in Fig. 1(A). A expanded portion of the spectrum with the transition assignments is displayed in Fig. 1(B).

width, enabling the true cross sections to be measured. Farmer et al. ${ }^{15}$ used the "Hook" technique to measure the band oscillator strengths of the $\gamma$ bands of $\mathrm{NO}$ including the $(3,0)$ band. Cieslik ${ }^{16}$ made low resolution measurements of $\mathrm{NO}$ at low pressure and used the equivalent width and curve of growth method to obtain the band oscillator strength of the $A(3)-X(0)$ band. Chan et al. ${ }^{17}$ used the high resolution dipole $(e, e)$ technique, which is not sensitive to the instrumental resolution. Laux and Kruger ${ }^{18}$ calculated ab initio the band oscillator strengths for the $\gamma$ band. Luque and Crosley ${ }^{19}$ also obtained the band oscillator strengths from the lifetime measurements after two photon excitation.

In this paper, we report the analysis of the $\beta(6,0)$ and the $\gamma(3,0)$ bands of NO near $198 \mathrm{~nm}$ by using the VUV FT spectrometer with synchrotron radiation for the background source. Accurate line positions and line strengths of the rotational lines have been retrieved from our FT spectra. The rotational term values of the $B^{2} \Pi_{r}(v=6)$ and $A^{2} \Sigma^{+}(v$ $=3$ ) levels have been evaluated and then fed to a least squares fitting program to obtain accurate molecular constants of these levels. Since the resolution of the present experiment is comparable to the Doppler widths, the determination of the band oscillator strengths of these bands has been performed using line by line measurements.

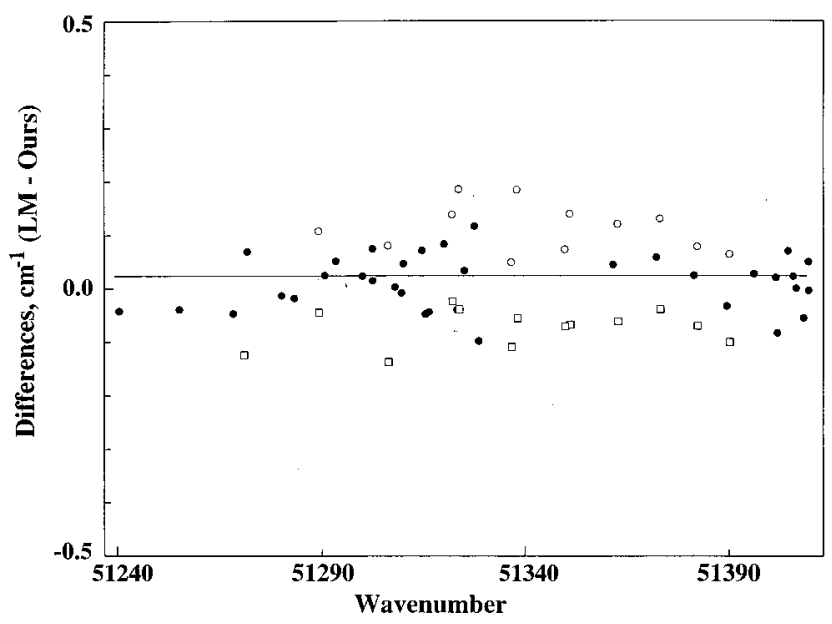

FIG. 2. Differences in the observed line positions of the $\beta(6,0)$ band between our results and Lagerqvist and Miescher (Ref. 13). The differences are given by the solid circles. For the lines separated $\Lambda$-doubling in this work are given by the open circles and squares. The solid horizontal line represents the average shift of $-0.019 \mathrm{~cm}^{-1}$.

\section{EXPERIMENT}

Details of the experimental procedures for recording high resolution FT spectra of NO between 160-198 nm have been described in our earlier publications. ${ }^{1-3}$ Only a brief description of the experimental conditions with respect to the recording of the $\beta(6,0)$ and $\gamma(3,0)$ bands will be given here. An absorption cell with optical path length of $7.54 \mathrm{~cm}$ was used, which was filled with 0.300 Torr of NO at $295 \mathrm{~K}$. The column density of $\mathrm{NO}$ in this experiment was $7.4 \times 10^{16}$ $\mathrm{mol} \mathrm{cm}{ }^{-2}$. A total of 236 scans, corresponding to above 10.7 hours of integration time, with a resolution of $0.06 \mathrm{~cm}^{-1}$, have been co-added. The signal-to-noise ratio in the continuum background was about 50 . The resonance line of $\mathrm{Hg}$ I at $184.8 \mathrm{~nm}$ was used as reference wave number in the previous papers. ${ }^{1,2}$ However, in this wavelength region we have no convenient absolute reference. From the calibration constant in the previous papers, we estimate the uncertainty in the absolute values as $0.02 \mathrm{~cm}^{-1}$. Relative uncertainties for the strong lines are better than $0.01 \mathrm{~cm}^{-1}$.

\section{RESULTS AND DISCUSSION}

The absorption spectra at around $195 \mathrm{~nm}$ were converted to optical depth by taking the logarithms of the intensity and fitting a smooth continuum to the regions between the lines. The absorption lines were fitted to Voigt profiles using the spectral reduction routine GREMLIN. ${ }^{20}$ The Voigt profile for the NO lines is a convolution of a Gaussian line shape due to Doppler broadening and a Lorentzian line shape arising from predissociation. Line parameters are determined through a nonlinear least squares iterative procedure that takes as free parameters both the Gaussian and the Lorentzian contributions as well as the line center and integrated intensity. However, in the fitting to retrieve line parameter, Gaussian component is sufficient to describe the line shape, because the upper levels of these band systems are located below the dissociation energy. The fitted profiles yielded an anoma- 
TABLE I. (a) Observed wave numbers of the $\gamma(3,0)$ band of NO. ${ }^{a}$ (b) Observed wave numbers of the $\beta(6,0)$ band of NO. ${ }^{\mathrm{c}}$

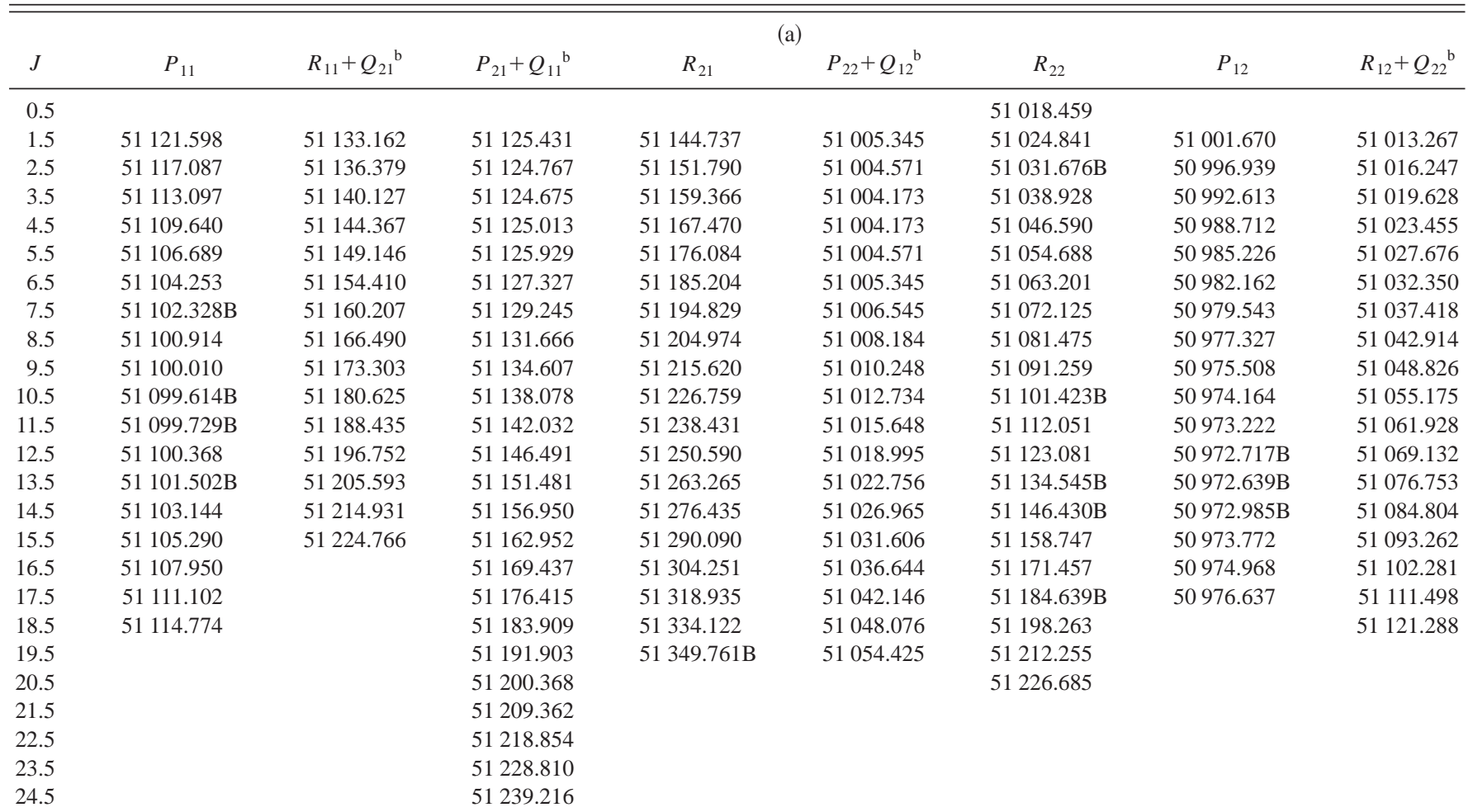

(b)

\begin{tabular}{|c|c|c|c|c|c|c|c|c|}
\hline$I$ & $R_{11}(J)_{e}$ & $R_{11}(J)_{f}$ & $P_{11}(J)_{e}$ & $P_{11}(J)_{f}$ & $Q_{11}(J)$ & $R_{22}(J)$ & $P_{22}(J)$ & $Q_{22}(J)$ \\
\hline 0.5 & \multicolumn{2}{|c|}{51409.450} & & & 51406.400 & & & \\
\hline 1.5 & \multicolumn{2}{|c|}{51409.504} & \multicolumn{2}{|c|}{51401.380} & 51404.430 & 51328.599 & & 51323.241 \\
\hline 2.5 & \multicolumn{2}{|c|}{51408.256} & \multicolumn{2}{|c|}{51396.073} & 51401.139 & 51327.484 & 51314.629 & 51320.017 \\
\hline 3.5 & \multicolumn{2}{|c|}{51405.677} & \multicolumn{2}{|c|}{51389.434} & & 51325.067 & 51307.998 & 51315.449 \\
\hline 4.5 & \multicolumn{2}{|c|}{51401.784} & \multicolumn{2}{|c|}{51381.476} & & 51321.365 & 51299.977 & 51309.609 \\
\hline 5.5 & 51396.035 & 51396.167 & \multicolumn{2}{|c|}{51372.242} & & 51316.345 & 51290.676 & 51302.486 \\
\hline 6.5 & 51390.036 & 51390.201 & \multicolumn{2}{|c|}{51361.656} & & 51310.054 & 51280.114 & \\
\hline 7.5 & 51382.222 & 51382.371 & 51349.727 & 51349.872 & & 51302.426 & 51268.248 & \\
\hline 8.5 & 51373.070 & 51373.240 & 51336.551 & 51336.710 & & 51293.449 & 51255.040 & \\
\hline 9.5 & 51362.680 & 51362.863 & 51321.962 & 51322.125 & & 51283.219 & 51240.543 & \\
\hline 10.5 & 51350.961 & 51351.169 & 51306.220 & 51306.438 & & 51271.631 & 51224.709 & \\
\hline 11.5 & 51337.916 & 51338.157 & 51289.093 & 51289.246 & & 51258.781 & 51207.593 & \\
\hline 12.5 & 51323.615 & 51323.840 & 51270.085 & 51270.925 & & 51244.584 & 51189.139 & \\
\hline 13.5 & 51307.980 & 51308.245 & 51251.014 & 51251.207 & & 51229.060 & 51169.374 & \\
\hline 14.5 & 51291.114 & 51291.343 & 51229.982 & 51230.226 & & 51212.256 & & \\
\hline 15.5 & 51273.019 & 51273.203 & 51207.654 & 51208.005 & & 51194.076 & & \\
\hline 16.5 & 51253.401 & 51253.738 & & & & 51174.602 & & \\
\hline 17.5 & & & & & & 51153.795 & & \\
\hline
\end{tabular}

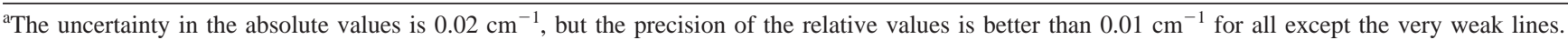

${ }^{\mathrm{b}}$ Transition line of the two branches are overlapped.

${ }^{c}$ The uncertainty in the absolute values is $0.02 \mathrm{~cm}^{-1}$, but the precision of the relative values is better than $0.01 \mathrm{~cm}^{-1}$ for all except the very weak lines.

lously large Gaussian contribution, with a full width at half maximum (FWHM) of $0.175 \mathrm{~cm}^{-1}$, as against the $0.12 \mathrm{~cm}^{-1}$ expected for Doppler width in our experimental conditions. This anomalous Gaussian width is considered to be due to drifts in alignment of the synchrotron beam and the external optics which cause very small wave number shifts over the long observation periods. ${ }^{21}$

\section{A. Line positions and molecular constants}

Figure 1(A) shows the Fourier transform spectrum of the $\beta(6,0)$ and $\gamma(3,0)$ bands near $195 \mathrm{~nm}$. As can be seen, the $\beta(6,0)$ band is considerably weaker than the $\gamma(3,0)$ band. The high $J$ lines of the $\gamma(3,0)$ band overlap heavily with the $\beta(6,0)$ band. Figure 1(B) is an expanded portion of the spectrum showing details of the rotational structure of the $\gamma(3,0)$ band. We observed 76 lines of the $\beta(6,0)$ band and these lines were assigned to six main branches $\left(P_{1}, Q_{1}, R_{1}, P_{2}\right.$, $Q_{2}$, and $\left.R_{2}\right)$. For the $\gamma(3,0)$ band, 224 lines belonging to 12 branches expected from a ${ }^{2} \Sigma-{ }^{2} \Pi$ transitions have been assigned for the first time. These lines are tabulated in (a) and (b) of Table I. Lagerqvist and Miescher ${ }^{13}$ had studied the 
TABLE II. The term values of the $A(3){ }^{2} \Sigma^{+}$and the $B(6){ }^{2} \Pi$ levels of $\mathrm{NO},{ }^{\mathrm{a}} \mathrm{cm}^{-1}$.

\begin{tabular}{|c|c|c|c|c|c|c|}
\hline \multirow[b]{3}{*}{$J$} & \multicolumn{2}{|c|}{$A(3)^{2} \Sigma^{+}$} & \multicolumn{4}{|c|}{$B(6){ }^{2} \Pi$} \\
\hline & \multirow{2}{*}{$\frac{F_{1}(J)}{e}$} & \multirow{2}{*}{$\frac{F_{2}(J)}{f}$} & \multicolumn{2}{|c|}{$F_{1}(J)^{2} \Pi_{1 / 2}$} & \multicolumn{2}{|c|}{$F_{2}(J)^{2} \Pi_{3 / 2}$} \\
\hline & & & $e$ & $f$ & $e$ & $f$ \\
\hline 0.5 & $51126.596(10)$ & $51130.467(01)$ & $51406.40(2)$ & $51406.41(1)$ & & \\
\hline 1.5 & $51130.452(02)$ & $51138.178(01)$ & $51409.45(1)$ & $51409.46(2)$ & $51448.15(1)$ & $51448.15(1)$ \\
\hline 2.5 & $51138.162(04)$ & $51149.758(12)$ & $51414.52(2)$ & $51414.53(2)$ & $51453.53(2)$ & $51453.53(2)$ \\
\hline 3.5 & 51 149.744(01) & $51165.190(01)$ & $51421.60(3)$ & $51421.65(1)$ & $51461.00(1)$ & $51461.00(1)$ \\
\hline 4.5 & $51165.176(02)$ & 51 184.482(09) & $51430.74(1)$ & $51430.79(1)$ & $51470.63(1)$ & $51470.63(1)$ \\
\hline 5.5 & $51184.465(06)$ & $51207.632(04)$ & $51441.87(3)$ & $51442.03(1)$ & $51482.41(2)$ & $51482.42(2)$ \\
\hline 6.5 & $51207.624(07)$ & $51234.639(07)$ & $51455.02(0)$ & $51455.24(3)$ & $51496.31(3)$ & $51496.31(3)$ \\
\hline 7.5 & $51234.625(03)$ & $51265.505(05)$ & $51470.26(0)$ & $51470.48(2)$ & $51512.35(1)$ & $51512.35(1)$ \\
\hline 8.5 & $51265.474(16)$ & $51300.209(04)$ & $51487.48(4)$ & $51487.73(3)$ & $51530.50(1)$ & $51530.51(1)$ \\
\hline 9.5 & $51300.179(52)$ & $51338.783(03)$ & $51506.79(1)$ & $51507.10(4)$ & $51550.74(1)$ & $51550.75(1)$ \\
\hline 10.5 & $51338.753(06)$ & $51381.205(08)$ & $51528.13(3)$ & $51528.42(3)$ & $51573.15(3)$ & $51573.15(3)$ \\
\hline 11.5 & $51381.172(04)$ & $51427.454(09)$ & $51551.56(4)$ & $51551.87(1)$ & $51597.69(3)$ & $51597.70(3)$ \\
\hline 12.5 & $51427.428(48)$ & $51477.584(04)$ & $51576.94(1)$ & $51577.30(1)$ & $51624.27(5)$ & $51624.28(5)$ \\
\hline 13.5 & $51477.552(05)$ & $51531.545(08)$ & $51604.41(2)$ & $51604.79(0)$ & $51653.04(3)$ & $51653.05(3)$ \\
\hline 14.5 & $51531.513(09)$ & $51589.358(08)$ & $51633.91(4)$ & $51634.37(4)$ & $51683.86(3)$ & $51683.87(3)$ \\
\hline 15.5 & $51589.311(13)$ & $51651.008(08)$ & $51665.52(3)$ & $51665.91(3)$ & $51716.82(3)$ & $51716.83(3)$ \\
\hline 16.5 & $51650.960(07)$ & $51716.499(16)$ & $51699.10(3)$ & $51699.60(3)$ & $51751.81(3)$ & $51751.83(3)$ \\
\hline 17.5 & $51716.464(06)$ & $51785.810(10)$ & $51734.77(3)$ & $51735.29(3)$ & $51788.93(3)$ & $51788.95(3)$ \\
\hline 18.5 & $51785.787(01)$ & $51858.992(17)$ & & & $51828.12(3)$ & $51828.14(3)$ \\
\hline 19.5 & $51858.953(04)$ & $51936.005(16)$ & & & & \\
\hline 20.5 & $51935.936(15)$ & $51916.813(26)$ & & & & \\
\hline 21.5 & $52016.765(15)$ & & & & & \\
\hline
\end{tabular}

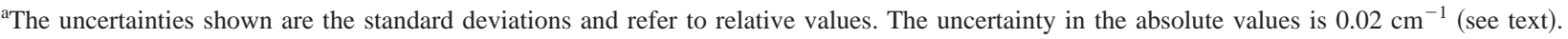

$\beta(6,0)$ band, and differences in the observed line positions (their values-our values) are plotted in Fig. 2. The agreement is excellent, $+0.019 \pm 0.092 \mathrm{~cm}^{-1}$. Our measurements have extended the assignment of rotational lines to $J=17.5$ of the $\beta(6,0)$ band and $J=21.5$ of the $\gamma(3,0)$ band. $\Lambda$-doubling of the $\beta(6,0)$ band has been observed in $R_{1}$ and $P_{1}$ branches.

The rotational term values of the $v=6$ level of the $B{ }^{2} \Pi_{r}$ state [upper state of the $\beta(6,0)$ band] and $v=3$ level of the $A^{2} \Sigma^{+}$state [upper state of the $\gamma(3,0)$ band] have been obtained by adding the term values of the $v=0$ level of the $X^{2} \Pi_{r}$ state to the wave numbers of the observed transition lines. The ground state rotational term values used are those of Amiot et al., ${ }^{22}$ for which the relative zero was set at the
$\Omega=1 / 2$ and $J=0.5$ level. The upper state term values obtained are averaged, and the results are listed in Table II, with the standard deviation shown as the uncertainty.

The effective Hamiltonian operator suitable for the description of the ${ }^{2} \Pi$ and ${ }^{2} \Sigma$ states can be found in Zare et $a .^{23}$ and Hougen. ${ }^{24}$ The matrix elements used in this work for the calculation of rotational energy levels are the same as those in Amiot et al. ${ }^{26}$ The parameters included in the description of the ${ }^{2} \Pi$ state are the band origin $T_{0}$; the rotational parameters $B$ and $D$; the spin-orbit parameters $A$ and $A_{D}$. For the ${ }^{2} \Sigma$ state: the band origin $T_{0}$; the rotational parameters $B$ and $D$; the spin-rotation constant $\gamma$. The rovibronic term values of the upper state of the $\beta(6,0)$ and $\gamma(3,0)$ bands were fitted to the parameters of the ${ }^{2} \Pi$ and ${ }^{2} \Sigma$

TABLE III. Molecular parameters of the $B^{2} \Pi v=6$ and $A^{2} \Sigma^{+} v=3$ levels of $\mathrm{NO}\left(\mathrm{cm}^{-1}\right){ }^{\text {a }}$

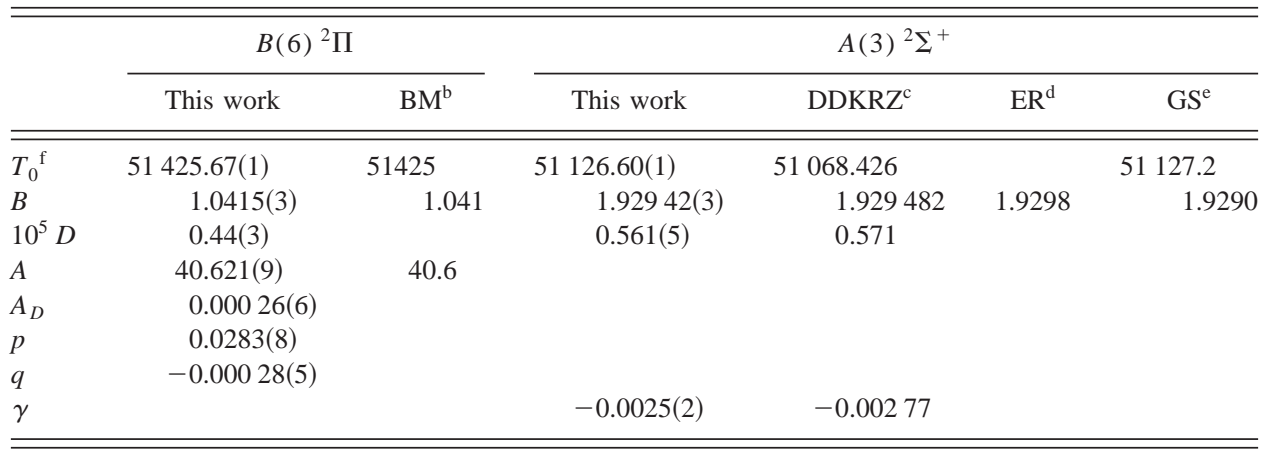

${ }^{a}$ Errors in parentheses are one standard deviation in units of the last significant figure quoted.

${ }^{\mathrm{b}} \mathrm{BM}$ : Barrow and Miescher (Ref. 25).

'DDKRZ: Danielak et al. (Ref. 12).

${ }^{\mathrm{d} E R}$ : Engleman and Rouse (Ref. 11).

eGS: Gerö and Schmid (Ref. 9).

${ }^{\mathrm{f}}$ The uncertainty in $T_{0}$ is dominated by the $0.02 \mathrm{~cm}^{-1}$ uncertainty in absolute wave numbers. 
TABLE IV. (a) Integrated cross sections of lines of the $\gamma(3,0)$ band of NO in units of $10^{-18} \mathrm{~cm}^{2} \mathrm{~cm}^{-1}$. (b) Integrated cross sections of lines of the $\beta(6,0)$ band of NO in units of $10^{-18} \mathrm{~cm}^{2} \mathrm{~cm}^{-1}$.

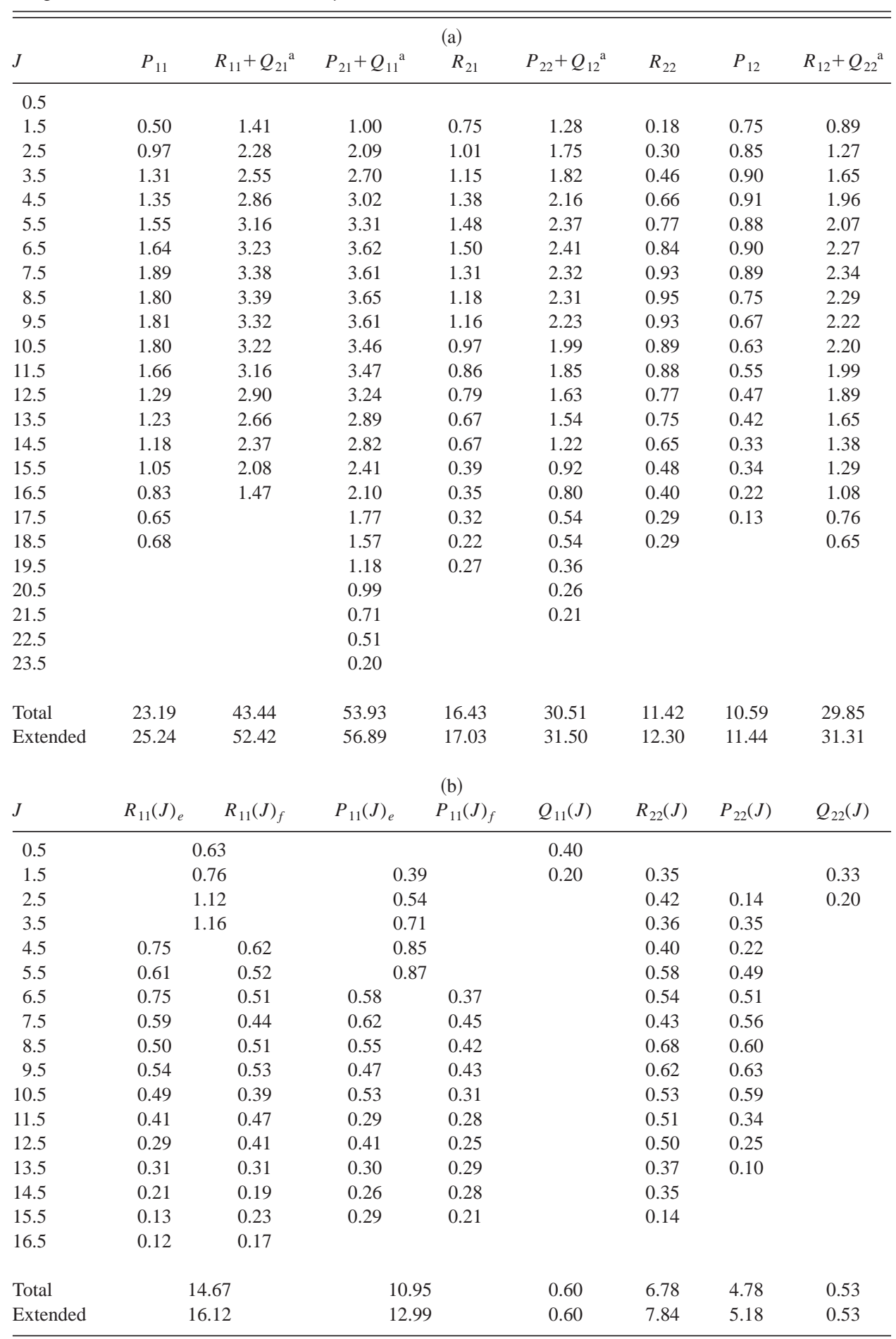

${ }^{a}$ Transition line of the two branches are overlapped. Total integrated cross section: $219.36 \times 10^{-18} \mathrm{~cm}^{2} \mathrm{~cm}^{-1}$. Extended integrated cross section: $238.13 \times 10^{-18} \mathrm{~cm}^{2} \mathrm{~cm}^{-1}$.

${ }^{\mathrm{b}}$ Transition line of the two branches are overlapped. Total integrated cross section: $38.31 \times 10^{-18} \mathrm{~cm}^{2} \mathrm{~cm}^{-1}$. Extended integrated cross section: $42.56 \times 10^{-18} \mathrm{~cm}^{2} \mathrm{~cm}^{-1}$.

state, respectively. Using a least squares package, the molecular parameters obtained for the $v=6$ level of the $B^{2} \Pi_{r}$ state and the $v=3$ of the $A^{2} \Sigma^{+}$state are listed in Table III. The molecular parameters determined by Engleman and Rouse, ${ }^{11}$ Danielak et al., ${ }^{12}$ and Barrow and Miescher ${ }^{25}$ are included for comparison. The apparent discrepancy between the band origin measured in this work and that of Danielak et al. ${ }^{12}$ arises from the use of different reference points in the $X^{2} \Pi$ state. In the ground state term value calculation, Danielak et al. ${ }^{12}$ used matrix elements in Table II of Amiot et al. ${ }^{26}$ which were different from those of Amiot et al. ${ }^{22}$ by $58.171 \mathrm{~cm}^{-1}$. This difference in term value is exactly equal 


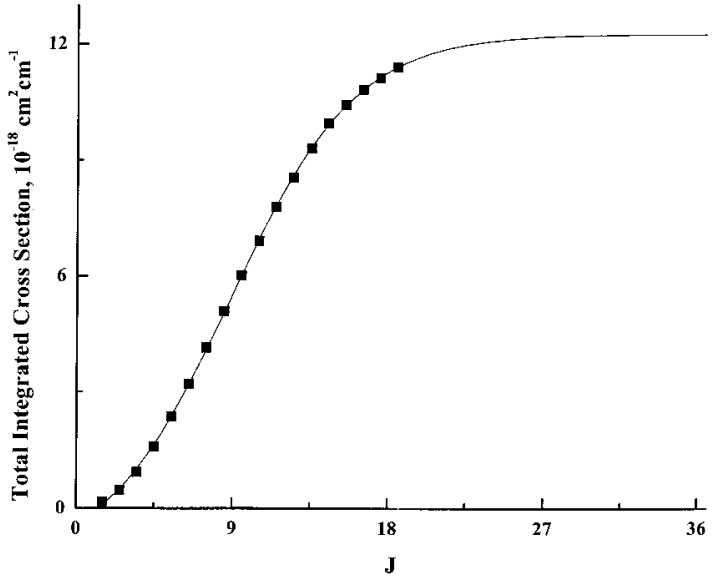

FIG. 3. Line by line total integrated cross-sections of the $R_{22}$ branch of the $\gamma(3,0)$ band.

to an amount $-\frac{1}{2} A+2 B+\frac{1}{2} p$ as listed in the matrix elements. Our molecular parameters were determined with high accuracy and the agreement between our values and those of earlier work is excellent.

\section{B. Band oscillator strength}

The band oscillator strengths are given by

$$
f\left(v^{\prime}, v^{\prime \prime}\right)=\frac{m c^{2}}{\pi e^{2}} \frac{1}{\tilde{N}\left(v^{\prime \prime}\right)} \int \sigma(\nu) d \nu,
$$

where $\tilde{N}\left(v^{\prime \prime}\right)$ is the fractional Boltzmann population of the absorbing vibrational level, and the integration of the cross section is performed over all the rotational lines belonging to the $\left(v^{\prime}, v^{\prime \prime}\right)$ band. In our retrieval of line parameters from the absorption spectrum, in addition to line positions, the integrated cross section of each line was also obtained. (a) and (b) of Table IV list the integrated cross sections of the rotational lines of these two bands. The integration of the cross sections of all observed rotational lines of the $\gamma(3,0)$ and $\beta(6,0)$ bands is equal to the sum of the integrated cross sections of all observed lines in (a) and (b) of Table IV, respectively. For the $\beta(6,0)$ band, the weak satellite branches from this $\Delta \Lambda=0$ transition were not observed. It is expected that the contribution of these satellite branches to the band oscillator strength should be insignificant. Observations of rotational lines are mostly limited to $J \leqslant 22.5$. The contributions of the higher $J$ lines are obtained by extending the total integrated cross sections to $J=36.5$. Figure 3 is a plot of the total integrated cross sections of the $R_{22}$ branch of the $\gamma(3,0)$

TABLE V. Band oscillator strength $\left(10^{-4}\right)$ of NO.

\begin{tabular}{llc}
\hline \hline & $\gamma(3,0)$ & $\beta(6,0)$ \\
\hline This work & 2.69 & 0.48 \\
Luque and Crosley (Ref. 19) & 3.00 & \\
Chan, Cooper, and Brion (Ref. 17) & 3.6 & 0.37 \\
Laux and Kruger (Ref. 18) & 3.41 & 0.431 \\
Cieslik (Ref. 16) & 3.0 & \\
Farmer, Hasson, and Nicholls (Ref. 15) & 2.4 & \\
Bethke (Ref. 14) & 3.60 & 0.462 \\
\hline \hline
\end{tabular}

band versus $J$. The solid line in the figure was obtained by fitting those experimental points and the extended contribution was estimated by extrapolating the line to $J=36.5$. The results of the extended contributions are also listed in (a) and (b) of Table IV. Table $\mathrm{V}$ gives the band oscillator strengths determined for the $\beta(6,0)$ and $\gamma(3,0)$ bands. Errors in the band oscillator strengths obtained by integration over all lines are estimated to be about $10 \%$ for the $\beta(6,0)$ band and $5 \%$ for the stronger $\gamma(3,0)$ bands, including uncertainties from extrapolation as well as those in the measured lines. Table V compares our values with those in the literature. Among all the band oscillator strength determinations, only ours were performed using line by line measurement with a resolution comparable to the Doppler width of the spectral lines. We notice that our value of the $\gamma(3,0)$ band is among the smallest of the published values, and suggest that our line by line measurement can identify accurately the transition lines belonging to the $\gamma(3,0)$ band and exclude any overlapping transition intensity of other bands. Our determined value for the $\beta(6,0)$ band is the largest among these values. We would like to postpone our discussion of band oscillator strengths of various band systems of NO in the wavelength region 160-195 $\mathrm{nm}$ until after the analyses of all these bands are completed.

\section{ACKNOWLEDGMENTS}

This work was supported in part by a NSF Division of Atmospheric Sciences Grant No. ATM-94-22854 to Harvard College Observatory, and by the NASA Upper Atmospheric Research Program under Grant No. NAG5-484 to the Smithsonian Astrophysical Observatory. We also acknowledge the Paul Instrument Fund of the Royal Society for the development of the VUV-FT spectrometer. The FTS measurements at the Photon Factory were made with the approval of the Photon Factory Advisory Committee (94G367). K.Y. thanks the Japan Society for the Promotion of Science for support. We would like to thank Dr. W. H. Parkinson for critical reading of this paper.

${ }^{1}$ K. Yoshino, J. R. Esmond, W. H. Parkinson, A. P. Thorne, J. E. Murray, R. C. M. Learner, G. Cox, A. S-C. Cheung, K. W-S. Leung, K. Ito, T. Matsui, and T. Imajo, J. Chem. Phys. 109, 1751 (1998).

${ }^{2}$ T. Imajo, K. Yoshino, J. R. Esmond, W. H. Parkinson, A. P. Thorne, J. E. Murray, R. C. M. Learner, G. Cox, A. S.-C. Cheung, K. Ito, and T. Matsui, J. Chem. Phys. 112, 2251 (2000).

${ }^{3}$ J. Rufus, K. Yoshino, J. R. Esmond, A. P. Thorne, J. E. Murray, T. Imajo, K. Ito, and T. Matsui, J. Chem. Phys. 115, 3719 (2001).

${ }^{4}$ E. Miescher and K. P. Huber, International Review of Science, Physical Chemistry Ser. 2, Vol. 3, Spectroscopy, edited by Ramsay (Butterworths, London, 1976).

${ }^{5}$ S. W. Leifson, Astrophys. J. 63, 73 (1926).

${ }^{6}$ G. Herzberg and L. G. Mundie, J. Chem. Phys. 8, 263 (1940).

${ }^{7}$ Y. Tanaka, J. Sci. Res. Inst. (Tokyo) 43, 160 (1949).

${ }^{8}$ G. Herzberg, A. Lagerqvist, and E. Miescher, Can. J. Phys. 34, 622 (1956).

${ }^{9}$ L. Gerö and R. Schmid, Proc. Phys. Soc. Jpn. 60, 533 (1948).

${ }^{10}$ M. Ogawa, Sci. Light (Tokyo) 3, 90 (1955).

${ }^{11}$ R. Engleman and P. E. Rouse, J. Mol. Spectrosc. 37, 240 (1971).

${ }^{12}$ J. Danielak, U. Domin, R. Kȩpa, M. Rytel, and M. Zachwieja, J. Mol. Spectrosc. 181, 394 (1997).

${ }^{13}$ A. Lagerqvist and E. Miescher, Helv. Phys. Acta 31, 221 (1958).

${ }^{14}$ A. Bethke, J. Chem. Phys. 31, 662 (1959).

${ }^{15}$ A. J. D. Farmer, V. Hasson, and R. W. Nicholls, J. Quant. Spectrosc. Radiat. Transf. 12, 627 (1972). 
${ }^{16}$ S. Cieslik, Bull. Cl. Sci., Acad. R. Belg. 63, 884 (1977).

${ }^{17}$ W. F. Chan, G. Cooper, and C. E. Brion, Chem. Phys. 170, 111 (1993).

${ }^{18}$ C. O. Laux and C. H. Kruger, J. Quant. Spectrosc. Radiat. Transf. 48, 9 (1992).

19 J. Luque and D. R. Crosley, J. Phys. Chem. 111, 7405 (1999).

${ }^{20} \mathrm{~J}$. W. Brault (private communication).

${ }^{21}$ P. M. Dooley, B. R. Lewis, S. T. Gibson, G. H. Baldwin, P. C. Cosby, J. L. Price, R. A. Copeland, T. G. Slanger, K. Yoshino, A. P. Thorne, and J. E. Murray, J. Chem. Phys. 109, 3856 (1998).
${ }^{22}$ C. Amiot, R. Basis, and G. Guelachvili, Can. J. Phys. 56, 251 (1978).

${ }^{23}$ R. N. Zare, A. L. Schmeltekopf, D. L. Albritton, and w. J. Harrop, J. Mol. Spectrosc. 48, 174 (1973).

${ }^{24}$ J. T. Hougen, NBS Monograph 115, Washington, DC, 1970.

${ }^{25}$ R. F. Barrow and E. Miescher, Proc. Phys. Soc., London, Sect. A 70, 219 (1957).

${ }^{26}$ C. Amiot, J-P. Maillard, and J. Chauville, J. Mol. Spectrosc. 87, 196 (1981). 